\title{
Improvement of quark propagator estimation through domain decomposition
}

\section{T. Burch* and C. Hagen}

Institut für Theoretische Physik, Universität Regensburg, D-93040 Regensburg, Germany.

E-mail: tommy.burch@physik.uni-r.de

Applying domain decomposition to the lattice Dirac operator and the associated quark propagator, we arrive at expressions which, with the proper insertion of random sources therein, can provide improvement to the estimation of the propagator. Schemes are considered for both open and closed (or loop) propagators. In the end, our technique for improving open contributions is similar to the "maximal variance reduction" approach of Michael and Peisa, but contains the advantage, especially for improved actions, of dealing directly with the Dirac operator. Using these improved open propagators for the Chirally Improved operator, we present preliminary results for the staticlight meson spectrum.

XXIVth International Symposium on Lattice Field Theory

23-28 July 2006

Tucson, Arizona, USA

${ }^{*}$ Speaker. 


\section{Introduction}

We present a method [1] for improving the estimation of quark propagators between different domains of the lattice. Our method turns out to be similar to that of "maximal variance reduction" (MVR) [2]. However, it contains the advantage that one can work directly with the chosen lattice Dirac operator. In the following sections, we present our method and some first results for staticlight mesons, where the Chirally Improved (CI) [3] , light quark propagator is calculated with the improved estimator.

\section{The method}

Decomposing the lattice into two distinct regions, the full Dirac matrix can be written in terms of submatrices

$$
M=\left(\begin{array}{ll}
M_{11} & M_{12} \\
M_{21} & M_{22}
\end{array}\right),
$$

where $M_{11}$ and $M_{22}$ connect sites within a region and $M_{12}$ and $M_{21}$ connect sites from the different regions. We can also write the propagator in this form:

$$
M^{-1}=P=\left(\begin{array}{ll}
P_{11} & P_{12} \\
P_{21} & P_{22}
\end{array}\right) .
$$

We consider a set of random sources, $\chi^{n}(n=1, \ldots, N)$, and the corresponding resultant vectors, $\eta^{n}=P \chi^{n}$, to derive useful expressions for our technique. Reconstructing the sources in one region, $\chi_{1}^{n}$, from the solution vectors everywhere, $\eta^{n}$, we may write

$$
\chi_{1}^{n}=M \eta^{n}=M_{11} \eta_{1}^{n}+M_{12} \eta_{2}^{n} .
$$

If we now apply the inverse of the matrix within one region, we have

$$
M_{11}^{-1} \chi_{1}^{n}=\eta_{1}^{n}+M_{11}^{-1} M_{12} \eta_{2}^{n}
$$

This can be solved for $\eta_{1}^{n}$ and substituted back into the naive estimator of the propagator between the two regions (repeated source indices, $n$, are summed over):

$$
\begin{aligned}
P_{12} & \approx \frac{1}{N} \eta_{1}^{n} \chi_{2}^{n^{\dagger}} \\
& \approx \frac{1}{N}\left[M_{11}^{-1}\left(\chi_{1}^{n}-M_{12} \eta_{2}^{n}\right)\right] \chi_{2}^{n^{\dagger}} \\
& \approx-\frac{1}{N}\left(M_{11}^{-1} M_{12} \eta_{2}^{n}\right) \chi_{2}^{n \dagger}
\end{aligned}
$$

where in the last line we eliminate the first term due to the fact that we expect $\lim _{N \rightarrow \infty} \chi_{1}^{n} \chi_{2}^{n \dagger}=0$. Writing out the full expression, we obtain

$$
\begin{aligned}
P_{12} & \approx-\frac{1}{N} M_{11}^{-1} M_{12} P \chi_{2}^{n} \chi_{2}^{n \dagger} \\
& =-M_{11}^{-1} M_{12} P_{22},
\end{aligned}
$$




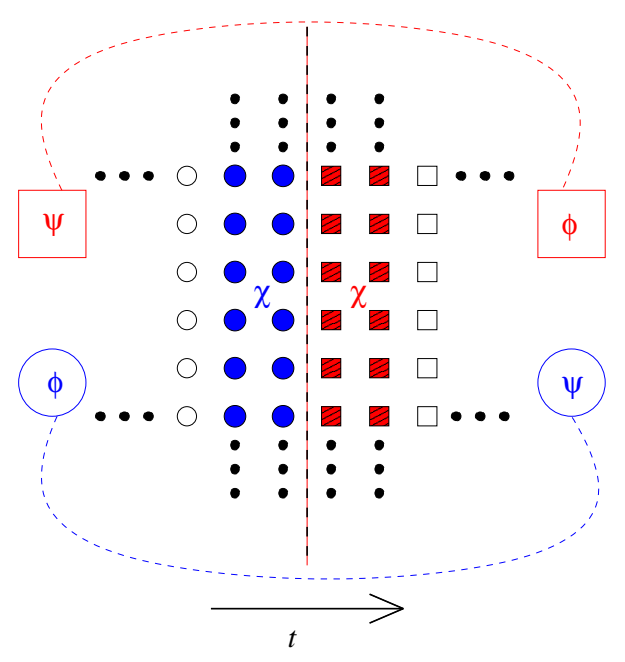

Figure 1: Depiction of one of the boundaries and the surrounding sources $(\chi)$ which we use to construct the two estimates of the CI quark propagator between regions of equal volume. Colors and shapes indicate which source region contributes to the signal in the resultant vectors. The $\psi$ 's are calculated using only one of the source regions, while the $\phi$ 's use both.

where the second line is an exact expression, showing that one can relate elements of different regions of $P=M^{-1}$ via the inverse of a submatrix of $M$. This is nothing new. After all, $P_{22}$ is the Schur complement of $M_{11}^{-1}$. But the lesson learned up to this point is that we need no sources in one of the two regions.

Looking again at Eq. (2.6), one can see that we need not make the approximation $P_{22} \approx$ $\frac{1}{N} P \chi_{2}^{n} \chi_{2}^{n \dagger}$. Instead, we can place the approximate Kronecker delta between the $M_{12}$ and $P_{22}$ :

$$
\begin{aligned}
P_{12} & \approx-\frac{1}{N} M_{11}^{-1} M_{12} \chi_{2}^{n} \chi_{2}^{n \dagger} P \\
& \approx-\frac{1}{N}\left(M_{11}^{-1} M_{12} \chi_{2}^{n}\right)\left(\gamma_{5} P \gamma_{5} \chi_{2}^{n}\right)^{\dagger} \\
& \approx-\frac{1}{N} \psi_{1}^{n} \phi_{2}^{n \dagger},
\end{aligned}
$$

where we have used the $\gamma_{5}$-hermiticity of the propagator. One can see from the form of the vector $\psi_{1}^{n}$ in the next to last line that we only need sources which "reach" region 1 via one application of $M$. Also, one can use all points in one region for the source and all points in the other region for the sink.

For our first attempt of using this method, we use equal volumes for the two regions and place sources next to the boundaries in both regions (see Fig. 1). Although this choice may not be ideal, we do perform inversions for each spin component separately (spin dilution) and, since our sources occupy all relevant time slices surrounding the boundaries, we actually obtain two independent estimates of the quark propagator between the two regions:

$$
-\frac{1}{N} \psi_{1}^{n} \phi_{2}^{n \dagger} \approx P_{12} \approx-\frac{1}{N} \gamma_{5} \phi_{1}^{n} \psi_{2}^{n \dagger} \gamma_{5}
$$




\begin{tabular}{lcc}
\hline oper. & $J^{P}$ & $\bar{Q} O\left(\Gamma, D_{i}\right) q^{\prime}$ \\
\hline$S$ & $0^{-}, 1^{-}$ & $\bar{Q} \gamma_{5} q^{\prime}$ \\
$P_{-}$ & $0^{+}, 1^{+}$ & $\bar{Q} \sum_{i} \gamma_{i} D_{i} q^{\prime}$ \\
$P_{+}$ & $1^{+}, 2^{+}$ & $\bar{Q}\left(\gamma_{1} D_{1}-\gamma_{2} D_{2}\right) q^{\prime}$ \\
$D_{ \pm}$ & $1^{-}, 2^{-}, 3^{-}$ & $\bar{Q} \gamma_{5}\left(D_{1}^{2}-D_{2}^{2}\right) q^{\prime}$ \\
\hline
\end{tabular}

Table 1: Static-light meson operators.

So our method is very similar to that of MVR, except for the fact that we can work directly with $M$, instead of $M^{\dagger} M$. This is better since it is less problematic to invert $M$ due to it having a better condition number than $M^{\dagger} M$ [2]. Also, the sources need only occupy enough time slices to connect them with the other region via $M$, rather than $M^{\dagger} M$. These are the same number of sources for Wilson-like operators, where $M^{\dagger} M$, like $M$, only extends one time slice. However, for many other improved operators (like CI) this can reduce the number of necessary source time slices by a factor of 2. On top of this, although the nature of the lattice Dirac operator may dictate what is the ideal domain decomposition, it does not otherwise restrict the choice of regions or the use of this method (e.g., it is even possible to use the Overlap operator).

For expressions and first results relevant to propagators which return to the same region (e.g., closed, or loop, propagators) we point the reader to our lengthier publication on the subject [1] and move on to our application for the open propagators.

\section{Static-light mesons}

For our meson source and sink operators, we use bilinears of the form:

$$
\bar{Q} O\left(\Gamma, D_{i}, \vec{D}^{2}, S\right) q,
$$

where $S$ is a gauge-covariant (Jacobi) smearing operator $[4$ ] and $\vec{D}$ is the covariant derivative. For our basis of light-quark spatial wavefunctions, we use three different amounts of smearing and apply 0,1 , and 2 covariant Laplacians to these:

$$
q^{\prime}=S_{8} q, \vec{D}^{2} S_{12} q, \vec{D}^{2} \vec{D}^{2} S_{16} q,
$$

where the subscript on the smearing operator denotes the number of smearing steps; all are applied with the same weighting factor of $\kappa_{s m}=0.2$. So we have a relatively narrow, approximately Gaussian distribution, along with wider versions which exhibit one and two radial nodes, due to the application of the Laplacians. We point out that, thus far, we have not altered the quantum numbers of the meson source since both the smearing and Laplacians treat all spatial directions the same (i.e., they are scalar operations). In order to create mesons of different quantum numbers, we use these light-quark distributions together with the operators shown in Table 1] (see, e.g., Ref. [⿰]𠃌 ]).

Inserting the estimated and static propagators in the meson correlators we have

$$
\begin{aligned}
C_{i j}(t) & =\left\langle 0\left|\left(\bar{Q} O_{j} q\right)_{t}\left(\bar{q} \bar{O}_{i} Q\right)_{0}\right| 0\right\rangle \\
& =\left\langle\sum_{x} \operatorname{Tr}\left[\frac{1+\gamma_{4}}{2} \prod_{i=0}^{t-1} U_{4}^{\dagger}(x+i \hat{4}) O_{j} P_{x+t \hat{4}, x} \bar{O}_{i}\right]\right\rangle_{\{U\}} .
\end{aligned}
$$



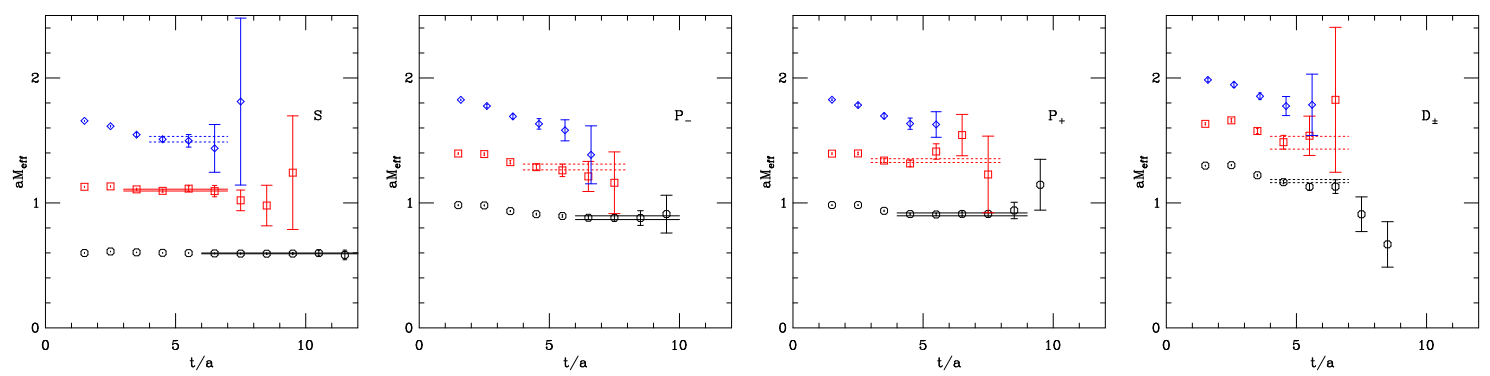

Figure 2: Effective masses for the static-light mesons on the quenched configurations. $a m_{q}=0.08, a^{-1} \approx$ $1330 \mathrm{MeV}, L \approx 1.8 \mathrm{fm}$. The horizontal lines represent $M \pm \sigma_{M}$ fit values for the corresponding time ranges. Dashed lines indicate fits for which we adjust the minimum time for systematic error estimates.

The static quark is propagated through products of links in the time direction and has a fixed spin $\left(1+\gamma_{4}\right) / 2$. The estimated propagator $P_{x+t \hat{4} . x}$ is of the form of Eq. (2.8). Thus, all points within region $1\left(N_{s}^{3} N_{t} / 2\right.$ of them) can act as the source location $x$, just so long as $t$ is large enough to have the sink location $x+t \hat{4}$ in region 2 . Note that we now have subscripts on the source and sink operators to denote which light-quark distribution is being used. We create all such combinations and thus have a $3 \times 3$ matrix of correlators for each of the operators in Table 1 .

Following the work of Michael [5] and Lüscher and Wolff [6], we use this cross-correlator matrix in a variational approach to separate the different mass eigenstates. We must therefore solve the generalized eigenvalue problem

$$
C(t) \vec{v}^{(k)}=\lambda^{(k)}\left(t, t_{0}\right) C\left(t_{0}\right) \vec{v}^{(k)}
$$

in order to obtain the following eigenvalues:

$$
\lambda^{(k)}\left(t, t_{0}\right) \propto e^{-t M_{k}}\left[1+O\left(e^{-t \Delta M_{k}}\right)\right],
$$

where $M_{k}$ is the mass of the $k$ th state and $\Delta M_{k}$ is the mass-difference to the next state. For large enough values of $t$, each eigenvalue should then correspond to a unique mass state, requiring only a single-exponential fit.

Variational approaches have seen much use recently in lattice QCD, especially for extracting excited hadron masses and we point the reader to the relevant literature in [7].

We create our cross-correlator matrices on two sets of gluonic configurations: 100 quenched and 74 dynamical, each with $12^{3} \times 24$ lattices sites. The quenched configurations have a lattice spacing of $a \approx 0.15 \mathrm{fm}\left(a^{-1} \approx 1330 \mathrm{MeV}\right)$ and a spatial extent of $L \approx 1.8 \mathrm{fm}$. The dynamical set [8] has 2 flavors of CI sea quarks (with $\left.M_{\pi \text {,sea }} \approx 500 \mathrm{MeV}\right), a \approx 0.115 \mathrm{fm}\left(a^{-1} \approx 1710 \mathrm{MeV}\right)$, and $L \approx 1.4 \mathrm{fm}$. We use 12 random spin-color vectors as sources for the light-quark propagator estimation. Spin-diluted, this gives us 48 separate sources for the inversions (one in the full volume, $\phi$, and two in the subregions, $\psi$; see Eqs. (2.7) and (2.8)). We perform inversions for 4 different quark masses: $a m_{q}=0.02,0.04,0.08,0.10$.

After extracting the eigenvalues, we check for single mass states by creating effective masses. A representative sample of these, along with single-elimination jackknife errors, are plotted against time in Fig. 2. In each case one finds values from the first three eigenvalues. The horizontal lines 

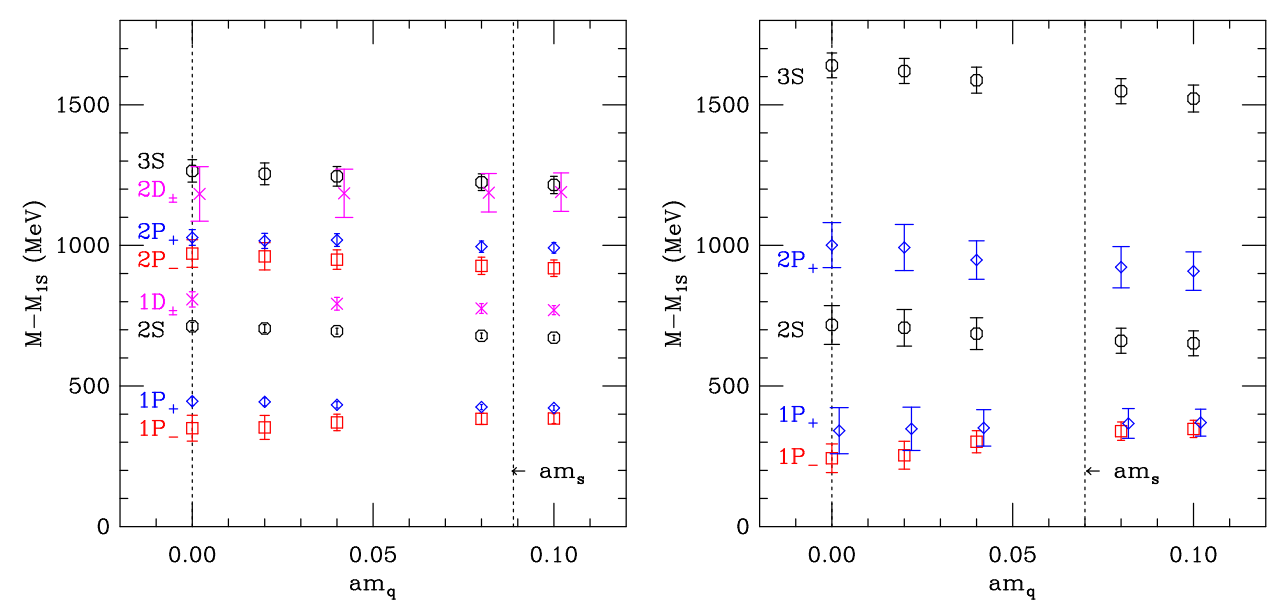

Figure 3: Physical mass splittings $\left(M-M_{1 S}\right)$ as a function of the quark mass for the quenched (left) and dynamical (right) lattices. The vertical lines denote the chiral limit $\left(m_{q} \rightarrow 0\right)$ and the strange quark mass $\left(m_{s}\right)$. The left-most values are the (linear) chiral extrapolations.

signify the $M \pm \sigma_{M}$ values which result from correlated fits over the corresponding range in time. We require that at least three effective mass points display a plateau (within errors) and that the eigenvectors remain constant (again, within errors) over the same range before we perform said fits.

Performing fits for all quark masses, we next take a look at the mass splittings $\left(M-M_{1 S}\right)$ as a function of the quark mass. These are plotted in Fig. 3, along with the chirally extrapolated results $\left(m_{q} \rightarrow 0\right)$. We use simple linear fits to perform these extrapolations.

In Table 1 we present the results for the chirally extrapolated ( $B$ mesons) and strange-quarkmass interpolated ( $B_{s}$ mesons) mass splittings. We include the statistical errors from the fits in the first set of parentheses. For fits where the effective-mass plateaus are not immediately clear (e.g., fits represented with dashed lines in Fig. 国), we move the minimum time of the fit range out by one to two time slices and observe the subsequent changes in $M \pm \sigma_{M}$, as compared to the previous values. The differences from the old values are reported as systematic errors; these appear in the second set of parentheses. For a discussion of these results, we refer the reader to our lengthier report [四].

One thing is clear though: due to the improvement of the light-quark propagator estimation, and our subsequent ability to use half the points of the lattice as source locations, we have greatly improved our chances of isolating excited heavy-light states. In an earlier study [9] of heavy-light mesons using wall sources on the same quenched configurations, we were barely able to see the $2 S$ state, let alone the excited states in any other operator channel. Also, there we used NRQCD for the heavy quark; this should only boost the signals since the heavy quark can then "explore" more of the lattice through its kinetic term. It is obvious, however, that we have much better signals now since we are able to see excited states in every channel $\left(2 S, 3 S, 2 P_{-}, 2 P_{+}\right.$, and $\left.2 D_{ \pm}\right)$on the quenched lattice. 


\begin{tabular}{lccc}
\hline state & $J^{P}$ & \multicolumn{2}{c}{$M-M_{1 S}(\mathrm{MeV})$ for $B / B_{s}$} \\
& & $N_{f}=0, L \approx 1.8 \mathrm{fm}$ & $N_{f}=2, L \approx 1.4 \mathrm{fm}$ \\
\hline $2 S$ & $(0,1)^{-}$ & $712(14) / 675(10)$ & $717(69) / 665(45)$ \\
$3 S$ & $(0,1)^{-}$ & $1265(40)\left({ }_{-130}^{+0}\right) / 1220(30)\left(_{-50}^{+20}\right)$ & $1640(44)\left({ }_{-200}^{+55}\right) / 1560(45)\left({ }_{-190}^{+35}\right)$ \\
\hline $1 P_{-}$ & $(0,1)^{+}$ & $350(46) / 384(20)$ & $243(51) / 330(34)$ \\
$2 P_{-}$ & $(0,1)^{+}$ & $971(49)\left({ }_{-90}^{+50}\right) / 923(30)\left({ }_{-60}^{+10}\right)$ & $-/-$ \\
\hline $1 P_{+}$ & $(1,2)^{+}$ & $446(15) / 424(10)$ & $341(82) / 363(55)$ \\
$2 P_{+}$ & $(1,2)^{+}$ & $1028(28)\left({ }_{-80}^{+160}\right) / 993(20)\left({ }_{-50}^{+130}\right)$ & $1001(80)\left({ }_{-20}^{+130}\right) / 930(75)\left({ }_{-80}^{+0}\right)$ \\
\hline $1 D_{ \pm}$ & $(1,2,3)^{-}$ & $808(27)\left({ }_{-90}^{+0}\right) / 773(17)\left({ }_{-80}^{+0}\right)$ & $-/-$ \\
$2 D_{ \pm}$ & $(1,2,3)^{-}$ & $1183(97)\left({ }_{-150}^{+130}\right) / 1188(68)\left({ }_{-80}^{+170}\right)$ & $-/-$ \\
\hline
\end{tabular}

Table 2: Mass splittings for our $B / B_{s}$ mesons. The first number in parentheses is the statistical error. The second set (if present) are the additional systematic errors which result from adjustments to the minimum time of the fit.

\section{Acknowledgments}

We would like to thank Christof Gattringer for many helpful discussions. We are also indebted to our colleagues in Graz - Christian B. Lang, Wolfgang Ortner, and Pushan Majumdar - for sharing their dynamical CI configurations with us. We also wish to thank Andreas Schäfer, without whose effort this interesting pursuit of ours would not be a paid one. Simulations were performed on the Hitachi SR8000 at the LRZ in Munich. This work is supported by GSI.

\section{References}

[1] T. Burch and C. Hagen, hep-lat/0607029.

[2] C. Michael and J. Peisa, Phys. Rev. D 58 (1998) 034506 [hep-lat/9802015].

[3] P. H. Ginsparg and K. G. Wilson, Phys. Rev. D 25 (1982) 2649; C. Gattringer, Phys. Rev. D 63 (2001) 114501 [hep-lat/0003005]; C. Gattringer, I. Hip, and C. B. Lang, Nucl. Phys. B 597 (2001) 451 [hep-lat/0007042].

[4] S. Güsken, et al., Phys. Lett. B 227 (1989) 266; C. Best, et al., Phys. Rev. D 56 (1997) 2743.

[5] C. Michael, Nucl. Phys. B 259 (1985) 58.

[6] M. Lüscher and U. Wolff, Nucl. Phys. B 339 (1990) 222.

[7] S. Sasaki, T. Blum, and S. Ohta, Phys. Rev. D 65 (2002) 074503 [hep-lat/0102010]; D. Brömmel, et al., Phys. Rev. D 69 (2004) 094513 [hep-ph/0307073]; A. M. Green, et al., Phys. Rev. D 69, 094505 (2004) [hep-lat/0312007]; T. Burch, et al., Phys. Rev. D 70 (2004) 054502 [hep-lat/0405006]; Phys. Rev. D 73 (2006) 017502 [hep-lat/0511054]; Phys. Rev. D 73 (2006) 094505 [hep-lat/0601026]; Phys. Rev. D 74 (2006) 014504 [hep-lat/0604019]; K. J. Juge, et al., hep-lat/0601029.

[8] C. B. Lang, P. Majumdar, and W. Ortner, Phys. Rev. D 73 (2006) 034507 [hep-lat/0512014].

[9] T. Burch, C. Gattringer, and A. Schäfer, Nucl. Phys. Proc. Suppl. 140 (2005) 347 [hep-lat/0408038]. 